13

\title{
Глубина выхода вторичных и фотоэлектронов из пленок CdTe с пленкой Ва
}

\author{
( Б.Е. Умирзаков, Д.А. Ташмухамедова, М.А. Турсунов, Ё.С. Эргашов, Г.Х. Аллаярова \\ Ташкентский государственный технический университет, \\ 100095 Ташкент, Узбекистан \\ e-mail: ftmet@mail.ru
}

Поступило в Редакцию 10 января 2019 г.

В окончательной редакции 10 января 2019 г.

Принято к публикации 20 фревраля 2019 г.

Впервые оценены зоны выхода $\lambda^{\prime}$ истинно-вторичных электронов и фотоэлектронов чистого СdTe и CdTe с пленкой Ва толщиной $\theta \leq 1$ монослоя. Показано, что с уменьшением работы выхода поверхности на $2 \mathrm{eV}$ значение $\lambda^{\prime}$ увеличивается в $1.2-1.3$ раза.

DOI: 10.21883/JTF.2019.07.47809.4-19

В настоящее время хорошо изучены влияния ионной имплантации на состав, электронную и кристаллическую структуры, эмиссионные и электрофизические свойства кристаллов $\mathrm{Si}, \mathrm{CdTe}, \mathrm{GaAs}, \mathrm{SiO}_{2}$ и $\mathrm{CaF}_{2}$ [1-3]. Однако изменения глубины выхода истинно-вторичных электронов (ИВЭ) и фотоэлектронов этих образцов при ионной бомбардировке являются мало изученными. Что касается глубины выхода ИВЭ образцов с субмонослойным покрытием различных элементов, то они практически не исследованы.

Под действием первичных электронов или фотонов в различных глубинах твердого тела возбуждаются электроны и часть из них эмиттируется в вакуум. При вторичной электронной эмиссии ИВЭ могут образоваться прямым потоком первичных электронов и неупругоотраженными электронами (НОЭ), а в случае фотоэмиссии фотоэлектроны образуются как прямым взаимодействием фотонов с электронами, так и взаимодействием этих же электронов с другими фотоэлектронами, с электронами твердого тела. Плотность возбужденных электронов и их глубина образования для различных материалов зависит от энергии первичных электронов (фотонов) и эффективности центров возбуждения приповерхностного слоя. Глубина выхода $\lambda$ этих электронов в вакуум в области энергии первичных электронов $E_{p}=100-500 \mathrm{eV}$ с ростом энергии увеличивается, а затем, начиная с $E=600-700 \mathrm{eV}$, не меняется. Последний называется зоной выхода $\lambda^{\prime}$ ИВЭ (фотоэлектронов) [4]. Для экспериментальной оценки глубины выхода электронов используются в основном методы эквивалентной подложки или диаграммы $\delta-\eta[4]$. Наши исследования показали, что когда $\eta$-подложки и пленки резко отличаются, то исследование зависимости $\delta(d)$ дает ценную информацию о глубине выхода истинно-вторичных и неупругоотраженных электронов в вакуум, где $\delta$ - коэффициент ИВЭ, $\eta-$ коэффициент $\mathrm{HOЭ}, d-$ толщина пленки. Тонкие пленки CdTe имеют особый интерес при создании оптических, электронных, фоточувствительных приборов, в частности солнечных элементов. Поэтому эмиссионные и оптические свойства, кристаллическая и электронная структуры CdTe и трехкомпонентные соединения на их основе в настоящее время хорошо изучены [4-7].

В настоящей работе впервые изучена глубина $\lambda$ и зона выхода $\lambda^{\prime}$ истинно-вторичных и фотоэлектронов $\mathrm{CdTe}$ и их изменения при адсорбции атомов Ва до одного монослоя. При одинаковых энергиях $E_{p}$ и $h v$ глубина выхода ИВЭ и фотоэлектронов существенно не отличается друг от друга. Поэтому здесь основные результаты приводились для вторичной электронной эмиссии.

\section{Методика эксперимента}

Поликристаллические пленки $\mathrm{CdTe}$ с толщиной 5.0-50.0 nm получены на поверхности Мо (111) методом газофазной эпитаксии. После технологических обработок (полировка ионами $\mathrm{Ar}^{+}$в сочетании с прогревом до $T \leq 900 \mathrm{~K})$ в сверхвысоком вакууме поверхность CdTe становится существенно гладкой, стехиометрический состав приближается к таковым для совершенных пленок CdTe [6]. После многократного распыления и прогрева поверхности подложки получены сплошные поликристаллические пленки $\mathrm{CdTe}$, начиная с толщины $5.0-6.0 \mathrm{~nm}$.

Исследования проводились при вакууме не хуже $10^{-6} \mathrm{~Pa}$ с использованием методов оже-электронной спектроскопии (ОЭС), ультрафиолетовой фотоэлектронной спектроскопии (УФЭС), спектроскопии характеристических потерь энергии электронов (СХПЭЭ) и измерением энергетических зависимостей коэффициентов вторичной электронной эмиссии (ВЭЭ). Для оценки величин $\lambda$ и $\lambda^{\prime}$ в основном применялся метод снятия зависимости $\delta(d)$. Морфологию и степень шероховатости поверхности изучали с помощью методов растровой электронной микроскопии (РЭМ) и атомно-силовой микроскопии (АСМ). Напыление Ва осуществлялось 
при вакууме не хуже, чем $10^{-5} \mathrm{~Pa}$. За один монослой принималась толщина пленки Ва, при которой значение работы выхода уменьшается до минимума ( $\delta$ увеличивается до максимума).

\section{Результаты экспериментальных исследований и их обсуждение}

Для исследования подготовлены в одинаковых условиях пленки $\mathrm{CdTe} / \mathrm{Mo} \mathrm{c} \mathrm{разной} \mathrm{толщиной} d=5.0-10.0$, $15.0,20.0$ и $50.0 \mathrm{~nm}$. Перед каждым исследованием поверхности пленок очищались прогревом при $T=900 \mathrm{~K}$ в течение $30-40 \mathrm{~min}$ в вакууме $P=10^{-6} \mathrm{~Pa}$. На рис. 1 приведены зависимости $\sigma\left(E_{p}\right)$ и $\eta\left(E_{p}\right)$ в области $E_{p}=100-1200 \mathrm{eV}$ чистого Мо (111) и Мо с пленкой $\mathrm{CdTe}$ толщиной $50.0 \mathrm{~nm}$ (где $\sigma$ - полный коэффициент ВЭЭ: $\sigma=\delta+\eta+r, r-$ коэффициент упру-

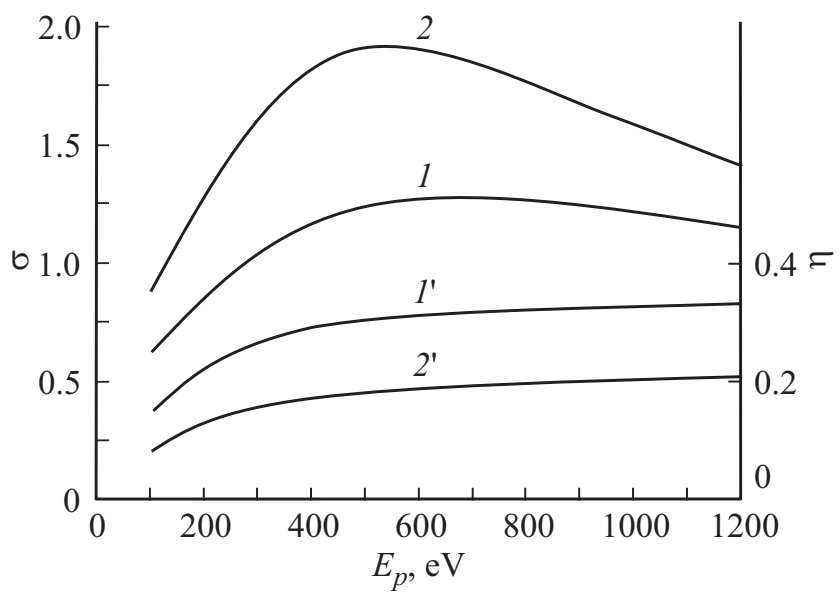

Рис. 1. Зависимости $\sigma$ (кривые 1,2$)$ и $\eta\left(1^{\prime}, 2^{\prime}\right)$ от $E_{p}$ для Мо $(1,2)$ и для пленки CdTe/Mo c $d=50.0 \mathrm{~nm}\left(1^{\prime}, 2^{\prime}\right)$.

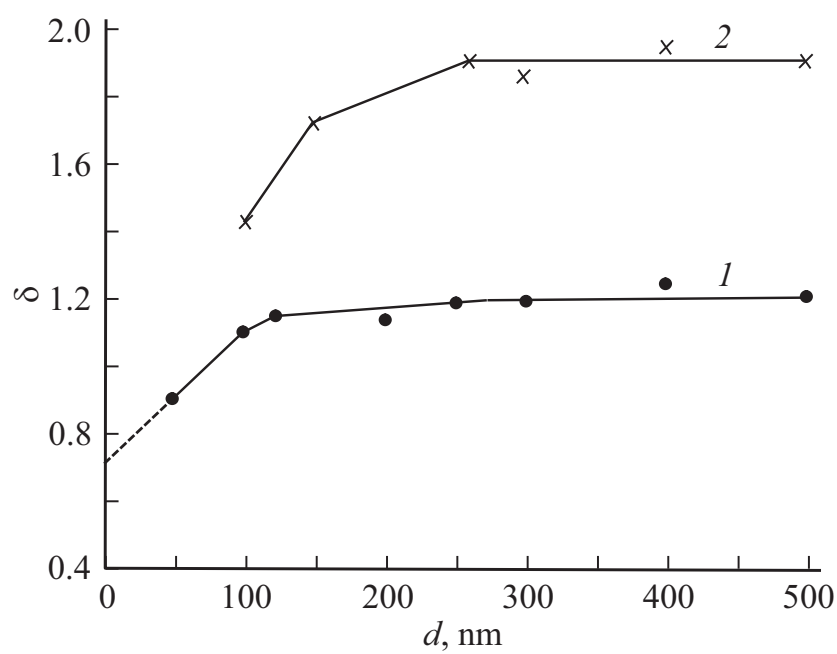

Рис. 2. Зависимости $\delta(d)$ для пленки $\mathrm{CdTe}$ до $(1)$ и после (2) напыления атомов Ва с толщиной $\sim 1$ монослой.

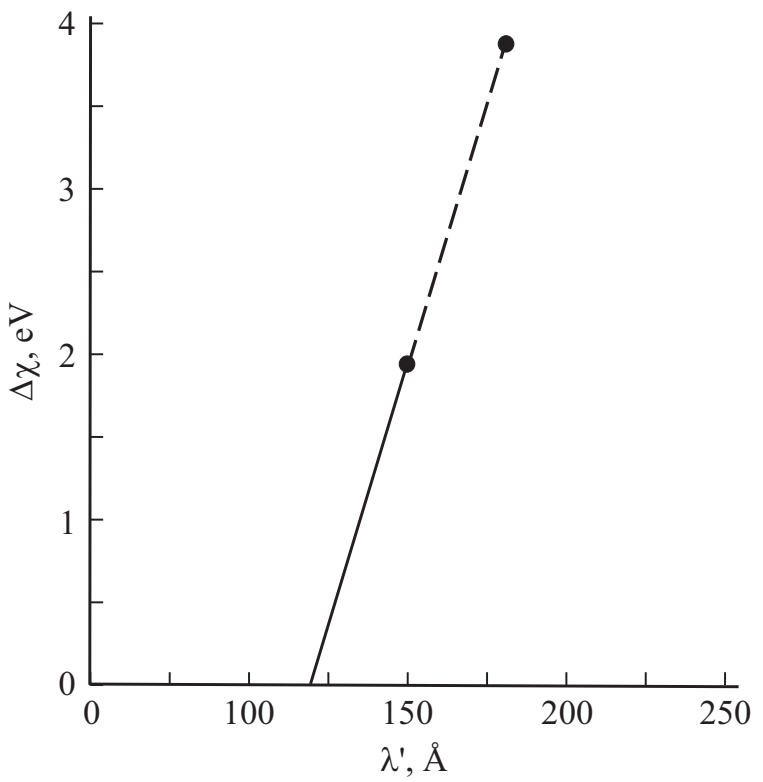

Рис. 3. Зависимость глубины зоны выхода ИВЭ от уменьшения работы выхода CdTe/Mo (111).

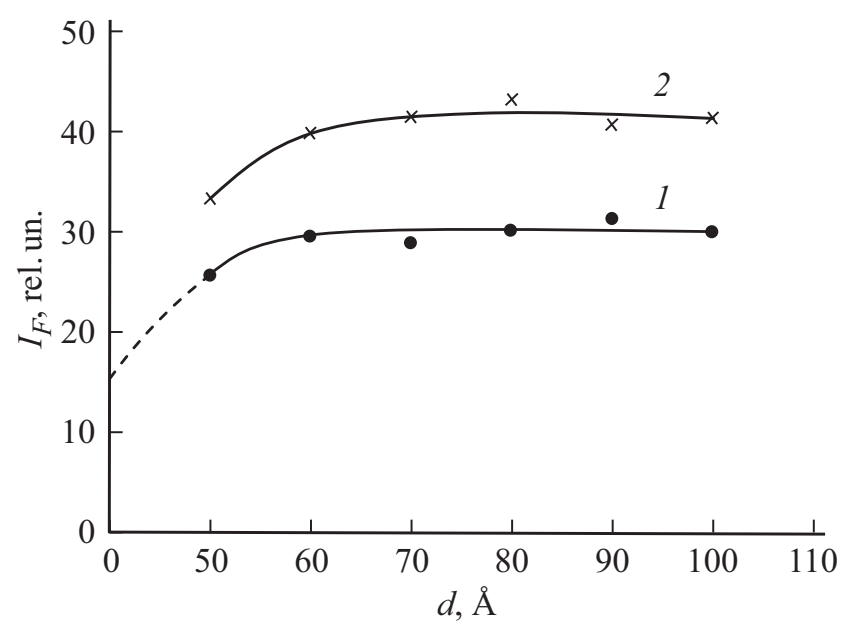

Рис. 4. Зависимость интенсивности фототока $I_{F}$ от толщины пленок $\mathrm{CdTe} / \mathrm{Mo} \mathrm{(111)} \mathrm{до} \mathrm{(1)} \mathrm{и} \mathrm{после} \mathrm{(2)} \mathrm{напыления} \mathrm{атомов}$ Ва с толщиной $\sim 1$ монослой.

го отраженных электронов). Видно, что значение $\sigma$ пленки проходит через максимум при $E_{p}=600-700 \mathrm{eV}$ $\left(\sigma_{m}=1.8\right)$, т. е. начиная с этой энергии глубина выхода ИВЭ $\lambda$ не меняется и равняется зоне выхода $\lambda^{\prime}[6]$. Значения $\eta$ для Мо и СdTe существенно отличаются друг от друга и практически остаются постоянными при $E_{p} \geq 700-800 \mathrm{eV}$. Поэтому для определения значения зоны выхода ИВЭ зависимость $\delta(d)$ снималась при $E_{p}=1000 \mathrm{eV}$ (рис. 2). Из рис. 2 (кривая 1) видно, что до $d=10.0-12.0 \mathrm{~nm}$ значение $\delta$ увеличивается линейно, затем скорость роста резко уменьшается и, начиная с $d=25.0 \mathrm{~nm}$, практически не меняется. Исходя из этого, можно полагать, что глубина зоны выхода ИВЭ $\lambda^{\prime}$ в случае СdTe составляет $\sim 10.0-12.0 \mathrm{~nm}$. Исследова- 
Зависимости зоны выхода ИВЭ от значений $\chi$ для CdTe

\begin{tabular}{c|c|l|l|c|c|c}
\hline Образец & $\begin{array}{c}\theta_{\mathrm{Ba}}, \\
\text { монослой }\end{array}$ & $\chi, \mathrm{eV}$ & $\Delta \chi^{*}, \mathrm{eV}$ & $E_{g}, \mathrm{eV}$ & $\lambda^{\prime}, \mathrm{nm}$ & $\lambda_{\mathrm{NERE}}, \mathrm{nm}$ \\
\hline \multirow{3}{*}{$\mathrm{CdTe}$} & 0 & 4.2 & 0 & 1.45 & 12.0 & 25.0 \\
& 0.5 & 3 & 1.2 & 1.45 & - & 25.0 \\
& 1.0 & 2.2 & 2.0 & 1.45 & 15.0 & 25.0
\end{tabular}

Примечание: ${ }^{*}-$ изменение величины $\chi$ относительно уровня вакуума.

ния зависимости $\delta-\eta$, снятые при $E_{p}=1000 \mathrm{eV}$ также показали, что для $\mathrm{CdTe} \lambda^{\prime}=10.0-12.0 \mathrm{~nm}$. Некоторое увеличение $\delta$ в интервале $d=12.0-25.0 \mathrm{~nm}$ может быть связано с выходом НОЭ с энергией меньше, чем $50 \mathrm{eV}$. C учетом этого можно полагать, что глубина выхода НОЭ при $E_{p}=1000 \mathrm{eV}$ составляет 20.0-25.0 nm. Напыление на поверхность пленок $\mathrm{CdTe}(d=10.0-50.0 \mathrm{~nm})$, пленок Ва с толщиной $\theta \leq 1$ монослоя приводило к увеличению коэффициента $\delta$ во всей исследуемой области $d$ (рис. 2, кривая 2). Наибольшее увеличение происходило при $\theta=1$ монослой. При этом значение еф уменьшается на $2 \mathrm{eV}$, из этой кривой видно, что резкий рост $\delta$ наблюдается в интервале $d=0-15.0 \mathrm{~nm}$, в интервале $d=15.0-25.0 \mathrm{~nm} \delta$ увеличивается всего лишь на $0.1-0.2$, а при $d \geq 25.0 \mathrm{~nm}$ практически не меняется. Из этого следует, что уменьшение $е \varphi$ на $\sim 2 \mathrm{eV}$ приводит к увеличению зоны выхода ИВЭ $\lambda^{\prime}$ на 4.0-5.0 nm, а глубина выхода НОЭ существенно не изменяется. Необходимо отметить, что при напылении изменение $е \varphi$ равно изменению сродства к электрону $\chi$ (ширина зоны проводимости).

В таблице приведены зонно-энергетические параметры и глубина зоны выхода ИВЭ пленки CdTe/Mo (111) с субмонослойной пленкой Ва разной толщины. Толщина $\mathrm{CdTe} \sim 50.0 \mathrm{~nm}$. Из таблицы видно, что при $\theta_{\mathrm{Ba}} \sim 1$ монослой значение $\chi$ уменьшается на $\sim 2 \mathrm{eV}$, a значения $E_{g}$ и глубина выхода неупругоотраженных электронов практически не меняется. При этом не меняются также глубина и эффективность образования вторичных электронов. Поэтому можно полагать, что увеличение коэффициента ВЭЭ и фотоэлектронов при уменьшении $\chi$ в основном обусловлено увеличением зоны выхода электронов. Из полученных результатов следует, что с уменьшением $\chi$ зона выхода $\lambda^{\prime}$ монотонно увеличивается. Если эта зависимость линейная, то можно оценить значение $\lambda^{\prime}$ при $\chi=0(\Delta \chi=-4.2 \mathrm{eV})$, которое составляет $\sim 17.0-18.0 \mathrm{~nm}$ (рис. 3). При этом ИВЭ выходят на уровень дна зоны проводимости.

Известно [8], что для твердых тел зависимость глубины выхода электронов от энергии электронов (фотонов) при $\lambda=5.0-10.0 \mathrm{~nm}$ проходит через минимум, т. е. при энергиях, меньших, чем $50-100 \mathrm{eV}$ с уменьшением энергии $E_{p}$ и $h v$, глубина выхода ИВЭ и фотоэлектронов вновь увеличивается. В качестве примера на рис. 4 приведена зависимость интенсивности фототока $I_{F}$ от $d$, измеренная при $h v=10.8 \mathrm{eV}$ для чистого $\mathrm{CdTe}$ и
$\mathrm{CdTe}$ с пленкой Ва толщиной $\sim 1$ монослой. Видно, что значение $I_{F}$ для чистого CdTе начиная с $d=6.0 \mathrm{~nm}$ практически не меняется, т. е. глубина выхода фотоэлектронов $\lambda=6.0 \mathrm{~nm}$. После уменьшени $\chi$ на $2 \mathrm{eV}$ значение $\lambda$ увеличивается до $8.0-9.0 \mathrm{~nm}$. Если учесть, что фотоэлектронная работа еф выхода $\mathrm{CdTe} \sim 5.65 \mathrm{eV}$, то при данной $h v$ из чистого CdTe в вакуум выходят только фотоэлектроны, а „вторичные“ электроны твердого тела, образующиеся под действием фотоэлектронов, не смогут выходить в вакуум. При уменьшении е $\varphi$ на $2 \mathrm{eV}$ наиболее быстрые вторичные электроны могут выходить в вакуум, что приводит к увеличению $\lambda$ и $I_{F}$.

\section{Заключение}

На основе экспериментальных исследований оценены глубина выхода $\lambda$ и зона выхода $\lambda^{\prime}$ ИВЭ и фотоэлектронов из пленок $\mathrm{CdTe}$ с субмонослойным покрытием Ва. Показано, что $\lambda^{\prime}$ для „чистого“ CdTe составляет $\sim 12.0 \mathrm{~nm}$. Уменьшение $\chi$ на $\sim 2 \mathrm{eV}$ приводит к увеличению $\lambda^{\prime}$ на $4.0-5.0 \mathrm{~nm}$. При этом значении $E_{g}$, глубина выхода НОЭ и глубины образования ИВЭ практически не изменяются. Поэтому можно полагать, что увеличение эмиссии истинно-вторичных и фотоэлектронов при уменьшении $\chi$ в основном обусловливается увеличением $\lambda^{\prime}$.

\section{Список литературы}

[1] Мурадкабилов Д.М., Ташмухамедова Д.А., Умирзаков Б.E. // Поверхность рентгеновские, синхротронные и нейтронные исследования. 2013. № 10. С. 5862. [Muradkabilov D.M., Tashmukhamedova D.A., Umirzakov B.E. // Journ. Surf. Investigation. $X$-ray, Synchrotron and Neutron Techniques. 2013. Vol. 7. N 5. P. 967-971.]

[2] Умирзаков Б.Е., Ташмухамедова Д.А., Рузибаева М.К., Таматов А.К., Донаев С.Б., Мавлянов Б.Б. // ЖТФ. 2013. Т. 83. Вып. 9. С. 146-149. [Umirzakov B.E., Tashmukhamedova D.A., Ruzibaeva M.K., Tashatov A.K., Donaev S.B., Mavlyanov B.B. // Techn. Phys. 2013. Vol. 58. N 9. P. 1383-1386. DOI: 10.1134/S1063784213090260]

[3] Эргашов Ё.С., Ташмухамедова Д.А., Джурабекова Ф.Г., Умирзаков Б.Е. // Известия РАН. Сер. физическая. 2016. T. 80. № 2. C. $157-159$. DOI: $10.7868 /$ S0367676516020071 [Ergashov Y.S., Tashmukhamedova D.A., Djurabekova F.G., Umirzakov B.E. // Bulletin of the Russian Academy of Sciences. Physics. 2016. Vol. 80. N 2. P. 138-140. DOI: $10.3103 / \mathrm{S} 1062873816020064]$

[4] Бронштейн И.М., Фрайман Б.С. Вторичная электронная эмиссия. М.: Наука, 1969. 305 с.

[5] Ткачук П.Н. // ФТТ. 2000. Т. 42. Вып. 11. С. 1961-1963.

[6] Krylyuk S.G., Korbutyak D.V., Kryuchenko Yu.V., Kupchak I.M., Vakhnyak N.D. // J. Alloys. Comp. 2004. Vol. 371. P. 142-145.

[7] Li Q., Jie W., Gu Z., Yang G., Wang T., Zhang J. // J. Cryst. Growth. 2004. Vol. 265. P. 159-164.

[8] Somorjai G. Chemistry in two dimensions: surfaces. NY: Cornell University Press. Ithaca, 1981. 552 p. 